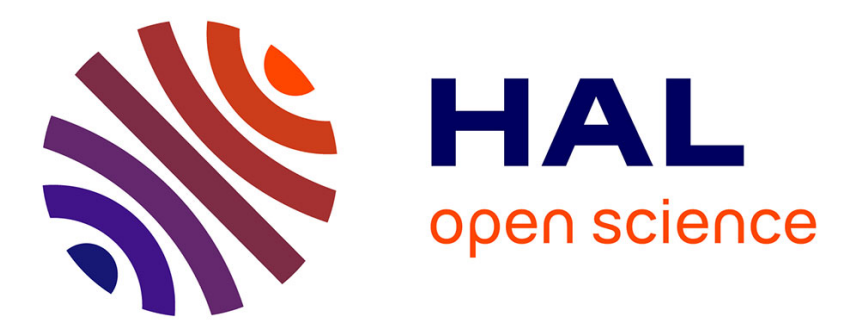

\title{
Insight: perspectives étiologiques et phénoménologiques dans la psychopathologie des désordres obsessionnels compulsifs
}

D. Belin, M.-L. Daniel, J. Lacoste, A. Belin-Rauscent, M. Bacconier, N. Jaafari

\section{To cite this version:}

D. Belin, M.-L. Daniel, J. Lacoste, A. Belin-Rauscent, M. Bacconier, et al.. Insight: perspectives étiologiques et phénoménologiques dans la psychopathologie des désordres obsessionnels compulsifs. Annales Médico-Psychologiques, Revue Psychiatrique, 2011, 169 (7), pp.420. 10.1016/j.amp.2011.06.003 . hal-00789296

\section{HAL Id: hal-00789296 \\ https://hal.science/hal-00789296}

Submitted on 18 Feb 2013

HAL is a multi-disciplinary open access archive for the deposit and dissemination of scientific research documents, whether they are published or not. The documents may come from teaching and research institutions in France or abroad, or from public or private research centers.
L'archive ouverte pluridisciplinaire HAL, est destinée au dépôt et à la diffusion de documents scientifiques de niveau recherche, publiés ou non, émanant des établissements d'enseignement et de recherche français ou étrangers, des laboratoires publics ou privés. 


\section{Accepted Manuscript}

Title: Insight: perspectives étiologiques et phénoménologiques dans la psychopathologie des désordres obsessionnels compulsifs

Authors: D. Belin, M.-L. Daniel, J. Lacoste, A.

Belin-Rauscent, M. Bacconier, N. Jaafari

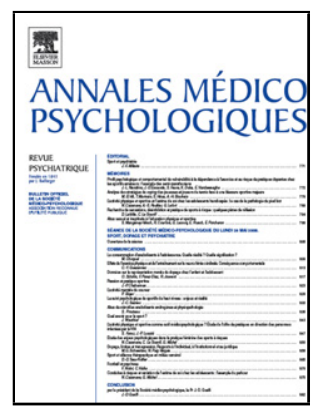

PII: S0003-4487(11)00162-4

DOI: doi:10.1016/j.amp.2011.06.003

Reference: AMEPSY 1344

To appear in: Annales Médico-Psychologiques

Please cite this article as: Belin D, Daniel M-L, Lacoste J, Belin-Rauscent A, Bacconier M, Jaafari N, Insight: perspectives étiologiques et phénoménologiques dans la psychopathologie des désordres obsessionnels compulsifs, Annales mediopsychologiques (2010), doi:10.1016/j.amp.2011.06.003

This is a PDF file of an unedited manuscript that has been accepted for publication. As a service to our customers we are providing this early version of the manuscript. The manuscript will undergo copyediting, typesetting, and review of the resulting proof before it is published in its final form. Please note that during the production process errors may be discovered which could affect the content, and all legal disclaimers that apply to the journal pertain. 


\section{Communication}

Insight : perspectives étiologiques et phénoménologiques dans la psychopathologie des désordres obsessionnels compulsifs

Insight: New vistas into an aetiological and phenomenological role in the psychopathology of obsessive compulsive disorders

D. Belin $^{\text {a }}$, M.-L. Daniel ${ }^{\text {a,b }}$, J. Lacoste ${ }^{\text {a,b }}$, A. Belin-Rauscent ${ }^{\text {a }}$, M. Bacconier ${ }^{\text {b }}$, N. Jaafari ${ }^{\text {a,b }}$

${ }^{a}$ INSERM, Équipe AVENIR, Psychobiologie des désordres compulsifs, Institut de Physiologie et de Biologie Cellulaires IPBC, UMR 6187 CNRS, Université de Poitiers, France

${ }^{b}$ Unité de recherche clinique intersectorielle Centre Hospitalier Henri Laborit, INSERM CIC-P 0802, CHU et faculté de médecine de Poitiers, France

Auteur correspondant: David Belin, INSERM, Équipe AVENIR, Psychobiologie des désordres compulsifs, Institut de Physiologie et de Biologie Cellulaires IPBC, UMR 6187 CNRS, Université de Poitiers, Bât. B36, Pôle Biologie Santé, 1, rue Georges Bonnet, BP 633, 86022 Poitiers Cedex, France

Tél. : 0549453401

Adresse email : david.belin@inserm.fr

Remerciements

David Belin est soutenu par un financement INSERM AVENIR, la fondation Fyssen, la

Fondation pour la Recherche Médicale, l'association Gilles de la Tourette, l'IREB,

l'université de Poitiers et le département de la Vienne.

Marie-Laure Daniel est interne en psychiatrie à Poitiers, actuellement en Master 2 de recherche dans l'équipe de David Belin, équipe AVENIR INSERM, Psychobiologie des désordres compulsifs, elle est soutenue par la Fondation pour la Recherche Médicale. Les auteurs remercient le Pr Senon pour son soutien aux réunions de réflexion qui ont lieu dans le service du centre hospitallier Henri Laborit.

\section{Résumé}

Dans le cadre du trouble obsessionnel-compulsif (TOC), les modèles psychopathologiques actuels, reposant sur un enchaînement dynamique par lequel la 
compulsion est émise en réponse à une forte anxiété, elle-même générée par l'obsession, n'intègrent pas la dimension de l'insight dans la survenue du trouble. En effet, bien que grandissante, la reconnaissance de l'implication de l'insight dans les désordres du spectre obsessionnel compulsif apparaît jusque-là ne s'être inscrite que dans une approche phénoménologique. Si cette perspective permet par exemple de rendre compte, sur le plan clinique, de l'apparent bon insight des patients souffrant d'un TOC, et qui sont généralement conscients de leurs symptômes, elle souffre de la discordance entre observations cliniques et évaluations psychométriques. Effectivement, plusieurs études mettent en évidence l'existence d'importants déficits d'insight chez certains de ces patients. Il semble donc nécessaire de revisiter le cadre théorique des désordres du spectre obsessionnel compulsif et de replacer le rôle d'insight dans la genèse de cette pathologie. Aussi proposons-nous un nouveau cadre conceptuel dans lequel l'insight pourrait jouer un rôle non seulement phénoménologique, mais également étiologique dans le TOC et les addictions. Pour cela, nous avons développé un nouveau modèle intégratif de l'insight basé sur l'intercomplémentarité de deux processus distincts, l'insight psychique et l'insight somatosensoriel, qui présente l'intérêt d'uniformiser la notion d'insight et d'en proposer une nouvelle échelle dimensionnelle. Cette nouvelle perspective pourrait permettre une meilleure compréhension de la psychopathologie des désordres du spectre obsessionnel compulsif et pourrait contribuer au développement de techniques thérapeutiques plus adaptées pour la prise en charge de ces patients.

Mots clés : Addictions ; Insight ; Phénoménologie ; Prise en charge ; Trouble obsessionnelcompulsif

\section{Abstract}

The current psychopathological models of obsessive/compulsive disorders (OCD) rely on a series of events whereby compulsions are expressed to self-medicate high anxiety levels generated by obsessions. These models do not take into account the implication of insight deficit in the development and expression of OCD. Although there is increasing interest in the role of insight deficits in obsessive/compulsive disorders, this interest has remained focused on the phenomenology of these disorders. If in apparent agreement with the clinical observation that OCD patients are overall well aware of their symptoms, such consideration of insights deficits restricted only to the phenomenology of obsessive disorders seems at odds both with other clinical dimensions than symptom awareness and psychometric measures. Indeed, several studies have demonstrated that some OCD patients do suffer from marked 
insight deficits. Therefore it seems necessary to reinvestigate the theoretical framework whereby insight may contribute to the psychopathology of obsessive disorders, obviously including OCD, but also drug addiction for instance. We therefore developed a model whereby insight deficits may not only contribute to the psychopathology of these disorders but also be involved in their aetiology. This model relies on a new theoretical framework that defines insight a general psychological process integrating two sub-processes, namely psychic insight and somatosensory insight, feeding into each other. This model, providing the basis for a new insight scale, has heuristic and predictive values with regards to new alternative etiological and phenomenological models of OCD and drug addiction. This novel theoretical framework may prove a valuable tool to better understand the psychopathology of, and develop new therapeutical strategies for, obsessive disorders.

Keywords: Addictions; Insight; Obsessive/compulsive disorders; Phenomenology; Therapeutical strategies;

Héritée de la psychiatrie française du XIX ${ }^{\mathrm{e}}$ siècle, la notion d’insight est intrinsèque à l'appréhension clinique de la psychopathologie [4]. Là réside sa richesse, mais là également sont ses limites. Si la légitimité de la question de l'insight n'est pas à discuter, le morcellement, au service de la pratique psychiatrique, de ce concept large en autant d'objets d’insight que de dimensions cliniques appréhendées, amène à une problématique théorique complexe : ne peut-on plus définir l'insight que par la mesure de ses déficits, aussi multiples que les objets auxquels le praticien se réfère dans le quotidien clinique $[9,10]$ ? En d'autres termes, l’insight, considéré à travers ses déficits, doit-il devenir un élément de la symptomatologie clinique lorsque ses plus récentes définitions ne s’inscrivent que dans une approche relative à la conscience, l'expérience, ou les objets diagnostiques, sans en proposer un concept uniforme [10] ? Sans considération de l'insight pour l'insight et par l'insight, la dichotomie de son acception en neurologie et en psychiatrie sera maintenue, chaque étude s'intéressera à sa définition de l'insight et la question de l'implication de l'insight dans le développement et l'expression des pathologies du cerveau restera, in fine, ouverte.

Appliquée aux désordres du spectre obsessionnel compulsif tels que les troubles obsessionnels compulsifs (TOC) ou les addictions, cette question prend toute sa dimension : l'insight dans les TOC relève-t-il de la conscience des symptômes [1,5,6,11], de la force de la croyance dans l'idée obsessionnelle [11], de la conscience des conséquences de sa pathologie, 
de la capacité à se ressentir [8] ? Selon une perspective plus large, pourquoi l'insight ne serait-il considéré que comme un facteur phénoménologique de ces pathologies psychiatriques ? C'est donc à la question de la place de l'insight dans les pathologies du spectre obsessionnel compulsif que nous nous intéresserons dans cette revue.

Nous proposons un nouveau cadre théorique de l'insight (figure 1) dans lequel il jouerait un rôle non seulement dans l'expression des désordres du spectre obsessionnel compulsif, mais également dans leur étiologie. Ainsi, comme illustré figure 1, l'insight résulterait de l'intégration de deux processus symétriques, avec une composante somatosensorielle (insight S, IS), organisée en couches successives d'intégrations structurées sur l'homéostasie, alors que la seconde composante serait psychique (insight $\mathrm{P}$, IP), structurée également en couches successives d’intégration basées, elles, sur l'homéostasie psychique. L'IS et l'IP sont associés de façon intime à une partie de la mémoire épisodique et autobiographique, considérée ici nécessaire notamment à l'introvision et au jugement par l'expérience, deux dimensions particulièrement importantes de l’insight.

Chacun des deux processus, IP et IS, contrôle étroitement le second, à chaque niveau des boucles d'intégration, de sorte qu'un déficit de l'un ou de l'autre, au niveau explicite ou non, se répercutera irrémédiablement sur le processus intégré qu'est l’insight. Ainsi, un même déficit d’insight appréhendé de façon clinique pourrait en réalité refléter différents types de déficits hybrides, résultant soit d'un déficit d'IS, amenant l'IP à traiter « sainement » des éléments $S$ anormaux, soit d'un déficit d'IP traitant de façon anormale les éléments $S$ sains produits par l'IS. Bien que pour le clinicien cette distinction présente peu d'intérêt dans la pratique, elle permet cependant, au sein d’un cadre général de l'insight, une attribution d'un déficit, selon une échelle dimensionnelle, à une altération de l’IS, de l’IP, ou des deux. Ainsi pouvons-nous envisager que les affections neurologiques associées à des déficits tels que l'anosognosie ou l'hallucination auditive dans la schizophrénie soient plus étroitement associées à un déficit de l’IS, particulièrement au niveau d'intégration de la représentation somatosensorielle, alors que les autres symptômes positifs de la schizophrénie, par exemple, seraient plus étroitement liés à un déficit de l’IP (figure 2).

Ce cadre théorique semble permettre une meilleure compréhension de la place de l'insight dans la phénoménologie du TOC (figure 3). Communément admise comme une séquence obsession - anxiété - compulsion (figure 3A) au sein de laquelle la pensée intrusive génère une forte anxiété à laquelle le patient répond par l'émission de compulsions anxiolytiques, la phénoménologie du TOC peut laisser une place importante à l’insight si elle est repensée dans un contexte où le moteur de l'obsession et/ou de la compulsion est le 
désordre anxieux (figure 3B). Dans ce contexte, au même désordre anxieux, le patient peut répondre soit par une séquence obsession/compulsion soit directement par une compulsion, selon qu'il présente un mauvais ou un bon IS. En d'autres termes, un patient présentant une bonne capacité à se ressentir pourrait développer la compulsion anxiolytique sans préalablement émettre une obsession, mais sans pour autant être capable de verbaliser la cause réelle de son comportement compulsif. Un patient avec un faible IS, lui, aurait développé l'obsession comme un processus de médication de ce faible IS, lui permettant de cristalliser son impression que « quelque chose ne va pas » en un objet mental, l'obsession, sur lequel il peut exercer un contrôle. Ce modèle place la nature du déficit d’insight au cœur des différences comportementales observées dans les populations de patients TOC.

La construction théorique que nous proposons permet également d'envisager une implication étiopathogénique de l'insight dans les désordres du spectre obsessionnel compulsif (figures 4 et 5). Cette perspective étend véritablement la conception de l'implication de l'insight dans la psychopathologie actuellement restreinte à la phénoménologie des troubles. Dans le cadre des TOC (figure 4), nous proposons qu’un déficit d'IS engendrerait chez le sujet prémorbide une incapacité à ressentir l'aspect anxiogène de certaines conditions environnementales. Ayant un déficit d'insight général, particulièrement attribuable au déficit d'IS, le sujet en phase prémorbide voit s'exercer une pression forte sur son état interne sans en avoir conscience. Après rupture des capacités adaptatives du patient, la pathologie s'installe, avec l'expression de la première obsession et l'établissement de cette sensation que «quelque chose ne va pas » souvent rapportée par le patient TOC. Notre modèle suggère que l'émergence de l'obsession s'accompagne d’une amélioration de l'IS. Ainsi, il y a matérialisation du désordre interne, que nous proposons représenter la pensée intrusive, et le patient peut, bien que malade, être conscient du désordre somatosensoriel, et, par la compulsion, agir dessus. La conscience du désordre ne préjuge pas d'une bonne attribution de ce désordre. En effet, le patient attribuera son anxiété à l’obsession et non pas à sa réelle cause, reflétant ainsi qu'il présente un déficit d’IP, lequel est également impliqué dans les répétitions des compulsions, considérées comme une incapacité à intégrer la conséquence de son action sur son environnement. Ainsi, bien que fluctuant en fonction de son état, selon l'échelle de mesure utilisée, l'insight du patient ne semblera pas nécessairement affecté lors d’évaluations cliniques. Cependant, nous suggérons que l’insight du patient est, de façon générale, amélioré par rapport à la situation prémorbide.

Dans les cas de patients TOC compulsifs, le gain d'IS ne passerait pas par l'obsession, mais résulterait d’une meilleure représentation somatosensorielle, permettant une réponse 
anxiolytique, via les compulsions, en réponse à cette représentation. Ces patients présenteraient cependant un plus faible IP que les patients obsessionnels car ils ne pourraient en aucun cas avoir conscience de cette nouvelle représentation somatosensorielle, ni lui attribuer leurs compulsions.

Ce modèle peut être étendu à d'autres désordres de la sphère obsessionnelle compulsive tels que l'addiction (figure 5). Dans ce cas, nous proposons que chez certains sujets, un déficit d’IS et un déficit d’IP contribuent respectivement à la recherche de sensations et l'impulsivité, deux traits étroitement liés à une vulnérabilité à développer une prise compulsive de drogue [2,3,7]. La recherche de sensations pourrait alors être considérée comme une quête du patient à se ressentir. Lors de la première exposition à une drogue, par exemple un stimulant, le sujet subirait un hyper insight iatrogène à la substance, il se ressentirait pour la première fois. Le sujet verrait alors cette « médication de l’IS » devenir un primum movens pour les prises ultérieures. Cependant, bien qu'une médication de l'IS fût envisagée chez ces patients, les effets psychobiologiques de l'utilisation de la substance s'accompagneraient d'une altération de l'IP, celle-ci contribuant à la myopie que présentent les patients par rapport aux conséquences négatives de leur addiction et la perte de contrôle sur leur prise.

Nous proposons un nouveau cadre conceptuel de l'insight permettant d'inclure cette dimension de la sphère psychique dans l'étiologie et la phénoménologie des désordres du spectre obsessionnel compulsif. Ce modèle, bien que conceptuel, permettrait une meilleure compréhension des phénomènes et objets relatifs de l’insight, étudiés selon différentes échelles et pour différentes pathologies. Cette nouvelle conception de l'insight permet également de s'affranchir de la notion que l'insight n'existe qu’à travers son déficit, dans la pathologie psychiatrique, lorsqu’il est défini par la conscience du trouble.

\section{Conflit d'intérêt: à compléter par l'auteur}

\section{Références}

[1] American Psychiatric Association. Diagnostic and Statistical Manual of Mental Disorders, DSM; 2000.

[2] Belin D, Berson N, Balado E, Piazza PV, Deroche-Gamonet V. High-novelty-preference rats are predisposed to compulsive cocaine self-administration. Neuropsychopharmacology 2011;36:569-79. 
[3] Belin D, Mar AC, Dalley JW, Robbins TW, Everitt BJ. High impulsivity predicts the switch to compulsive cocaine-taking. Science 2008;320:1352-5.

[4] Billod E. Discussion sur les aliénés avec conscience. Ann Med Psychol 1870;3:264-81.

[5] Bourgeois ML. La conscience du trouble en psychiatrie. II. Travaux empiriques actuels. La mesure de l’insight. Ann Med Psychol 2000;158:209-24.

[6] Bourgeois ML, Koleck M, Roig-Morrier R. Conscience du trouble chez 100 malades hospitalisés en psychiatrie. Ann Med Psychol 2002;160;444-50.

[7] Franques P, Auriacombe M, Tignol J. Addiction and personality. Encéphale 2000;26:6878.

[8] Goldstein RZ, Craig AD, Bechara A, Garavan H, Childress AR, Paulus MP, Volkow ND. The neurocircuitry of impaired insight in drug addiction. Trends Cogn Sci 2009;13:372-80.

[9] Marková IS, Berrios GE. The "object” of insight assessment: relationship to insight “structure”. Psychopathology 2000;34:245.

[10] Marková IS. L’insight en psychiatrie. Traduction par Jaafari N. Paris: Doin; 2009.

[11] Marková IS, Jaafari N, Berrios GE. Insight and obsessive-compulsive disorder: a conceptual analysis. Psychopathology 2009;42:277-82. 

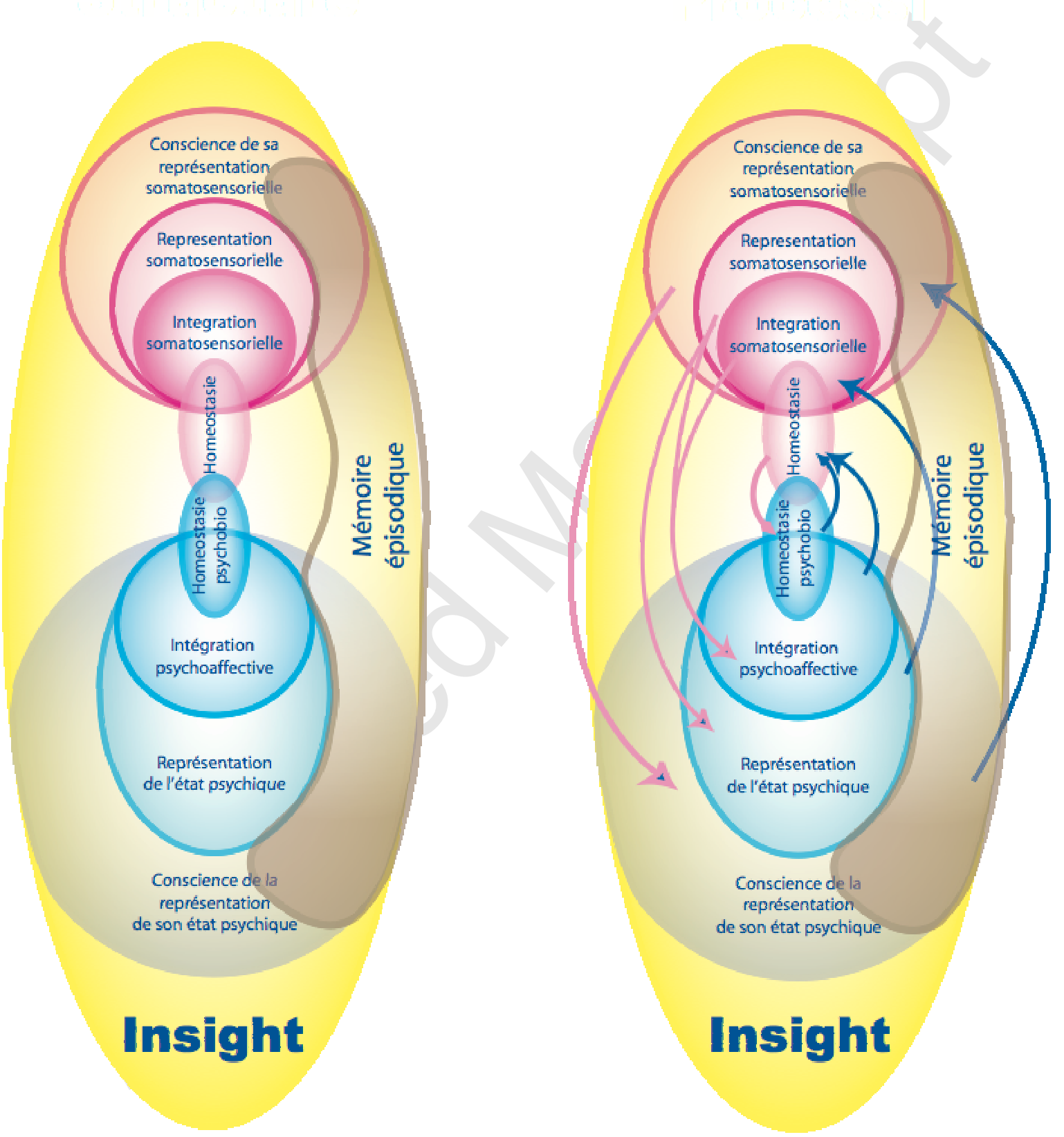


\section{Figure 1 : structure et processus de l'insight}

À gauche, l'insight est comme un tout décomposé en éléments simples en se référerant à un modèle bipolaire comprenant un insight somatosensoriel et un insight psychique. À partir de deux boucles initiales, homéostasie somatique et homéostasie psychique, structuration en couches d'intégration successives, de manière symétrique : à partir des informations somatosensorielles, représentation par le sujet de son état somatosensoriel, puis à un niveau supérieur, conscience de la représentation de ses sensations ; de même, intégration psychoaffective donnant lieu à des représentations de l'état psychique, et à la conscience de ces représentations. De plus, mémoire autobiographique et épisodique faisant lien entre conscience des états somatiques et des états psychiques, et exerçant une influence sur la manière dont l'individu prend subjectivement conscience de son expérience vécue.

À droite, interdépendance des deux systèmes de structuration : influences mutuellement exercées de part et d'autre, avec l'insight comme résultat final, complexe, de l'interaction des différentes boucles et processus en jeu.

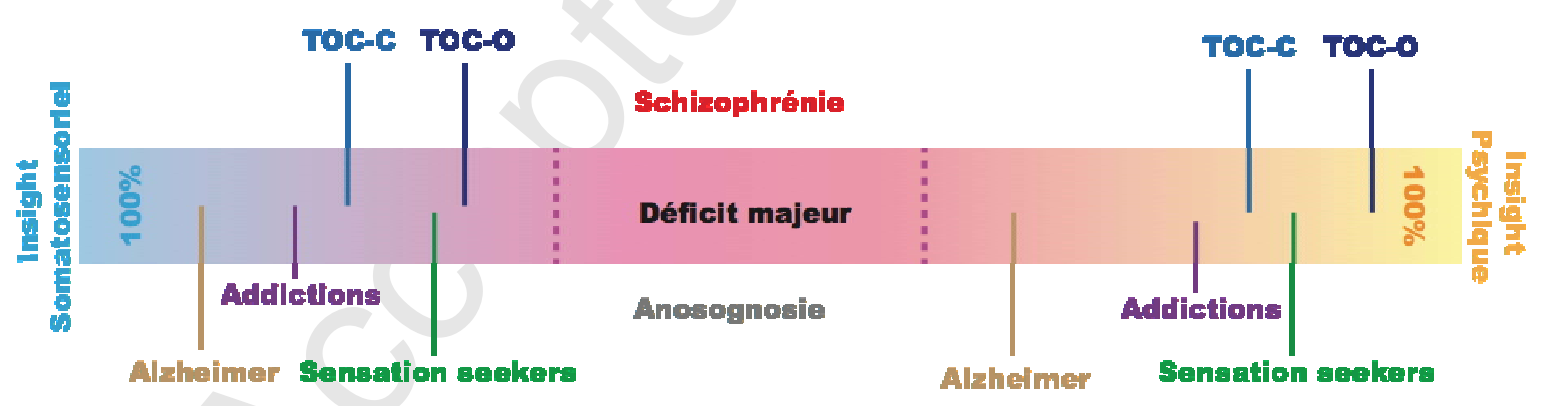

Figure 2 : échelle dimensionnelle de l'insight

Pour un déficit apparent d'insight, attribution sur l'échelle à une ou deux dimensions en cause: déficit d'insight somatosensoriel (IS à gauche, extrémité gauche pour un bon niveau d'IS) et/ou déficit d'insight psychique (IP à droite, extrémité droite pour un bon niveau d'IP). Ainsi, à gauche, association de certaines pathologies, telles que les TOC, à un déficit d’IS ; également, des traits de personnalité comme recherche de sensation. À droite, altération de l'IP prédominant par exemple dans la maladie d'Alzheimer (déficit mnésique et trouble du jugement). Dans l'addiction représentée des deux côtés, contribution mixte et variable des déficits de l'IS et de l'IP (défaut à se ressentir d'une part et myopie des conséquences néfastes du comportement d'autre part, par exemple). Au milieu, en cas d'altération majeure de l'un des processus, du fait de leur interdépendance, atteinte de l'autre concomitante comme dans la schizophrénie et l'anosognosie. 


\section{A}

B
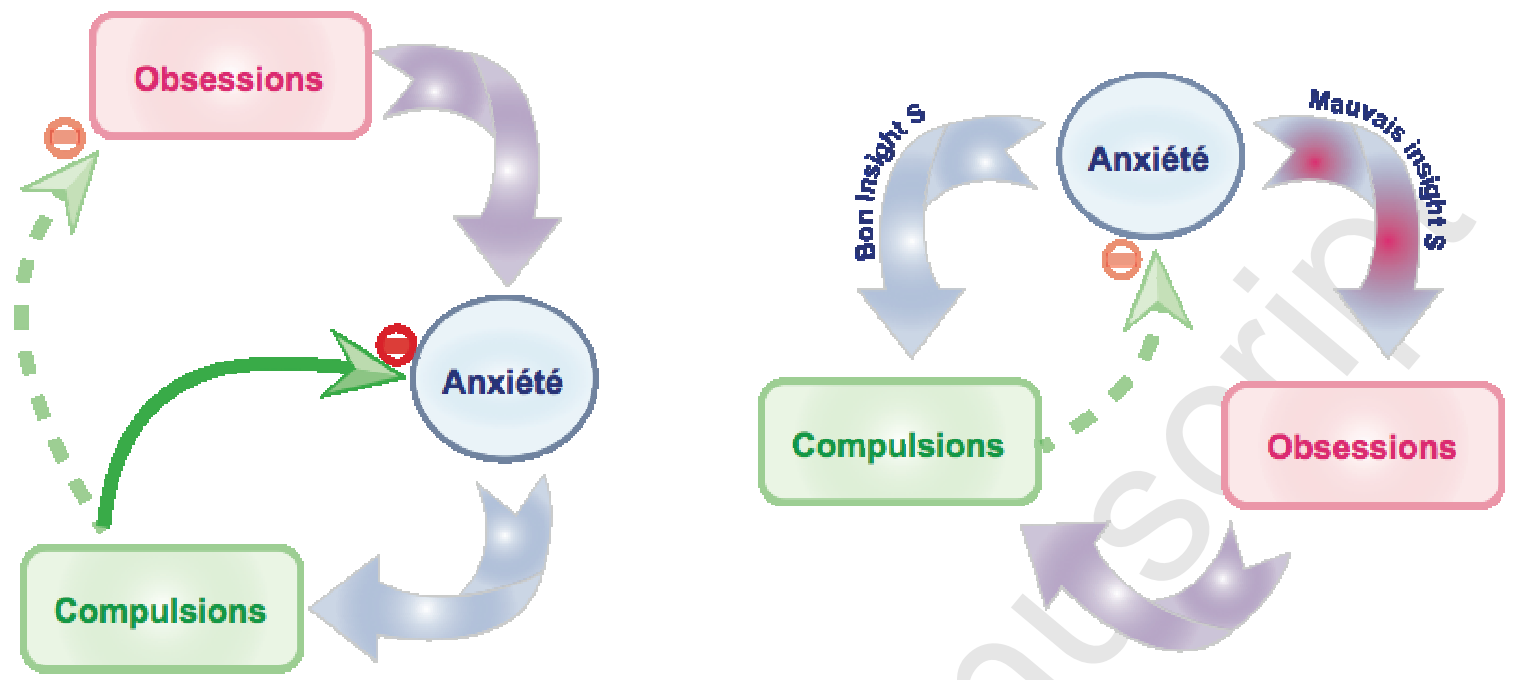

Figure 3 : modèle phénoménologique des troubles obsessionnels compulsifs

À gauche, séquence psychopathologique habituelle : obsession à l'origine de l'anxiété ; émission du comportement compulsif comme réponse à cette anxiété, qui en conséquence décroît.

À droite, modèle alternatif proposé : désordre anxieux à l'initiation de la séquence des troubles. Si bon IS, émergence de la compulsion et effet anxiolytique direct, mais pas de prise de conscience de la cause du comportement (faible IP). Si faible IS, recours à l'obsession comme objet mental matérialisant aux yeux du sujet la cause de son mal-être jusque-là mal déterminé (meilleur IP), puis contrôle possible pour l'individu par la compulsion. 


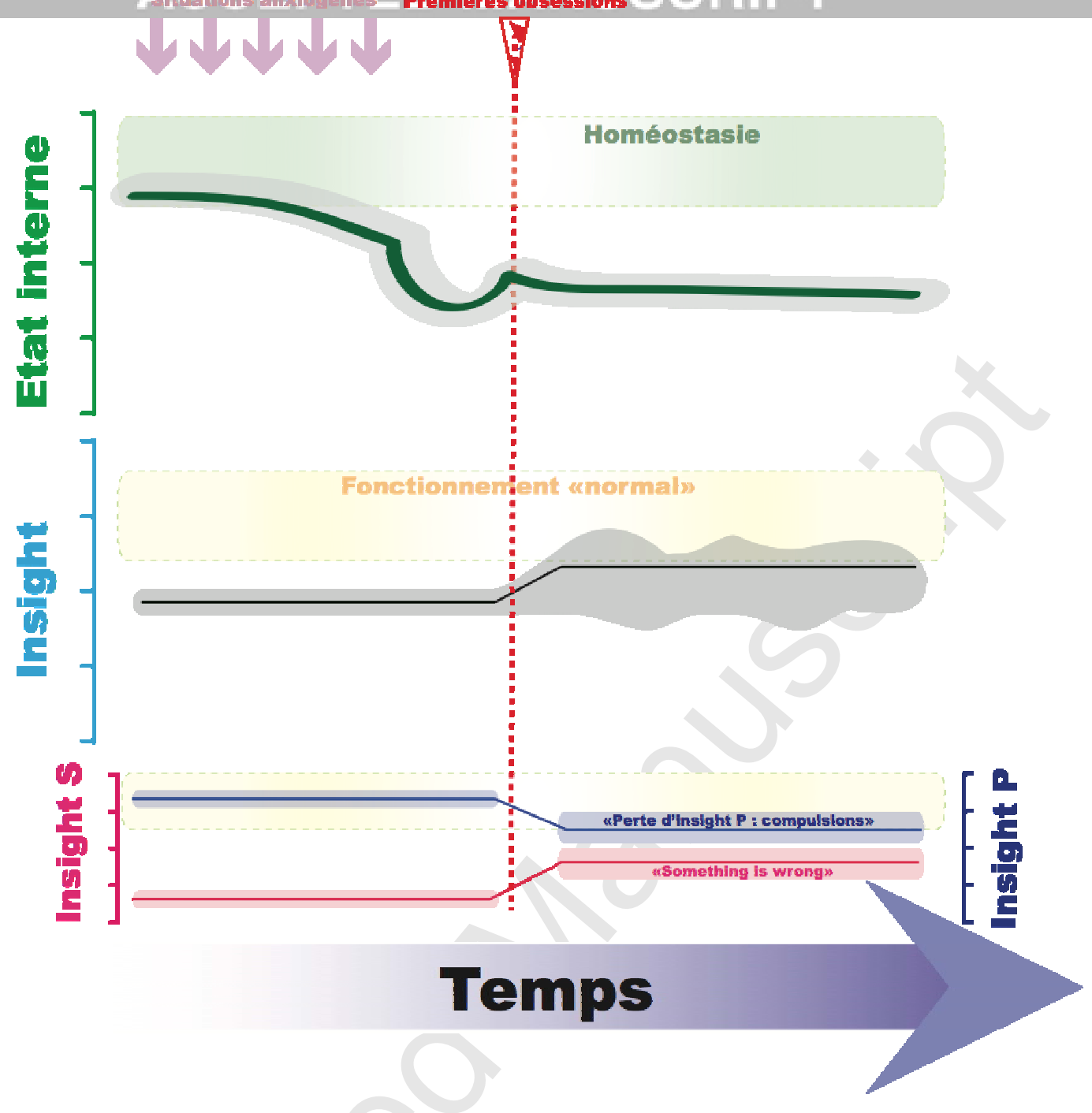

Figure 4 : perspective étiologique de l'insight dans les troubles obsessionnels compulsifs

Représentation schématique au fil du temps de l'implication potentielle de l'insight dans la survenue des TOC : évolutions parallèles de l'état interne, de l'insight et des insight $\mathrm{S}$ et insight $\mathrm{P}$. En haut, état interne (ligne verte) perturbé chez le sujet exposé à des situations anxiogènes. Au milieu, déficit d'insight (ligne noire) plus important avant développement de la pathologie. En bas, composante somatosensorielle de l'insight (ligne rose) : initialement déficit notable d'insight S, ensuite en partie compensé par la survenue des premières obsessions ("something is wrong »). En bas, composante psychique de l'insight (ligne bleue): perte d'insight $\mathrm{P}$ en lien avec le développement et l'extension des compulsions. 


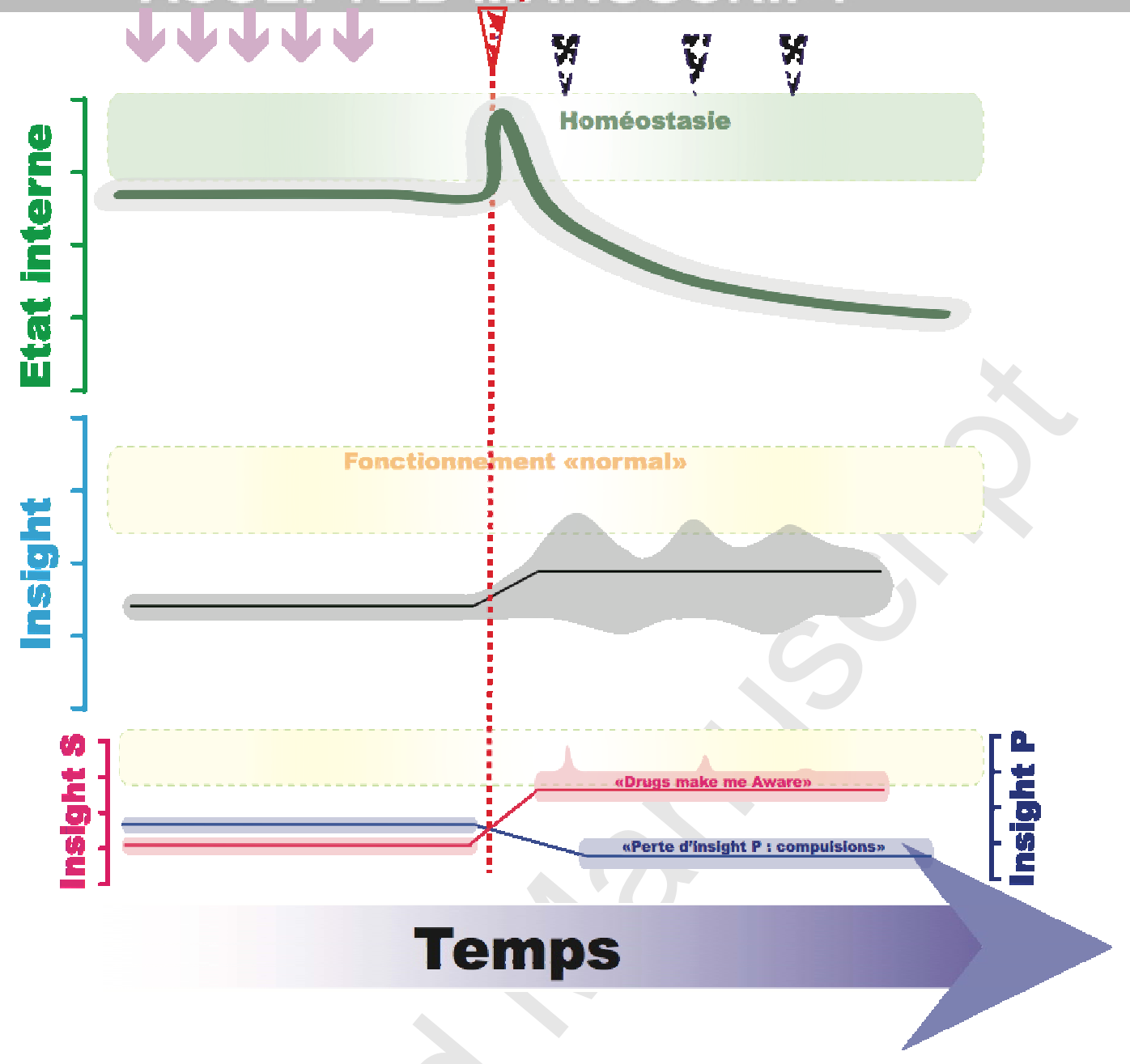

Figure 5 : perspective étiologique de l'insight dans l'addiction

Représentation schématique au fil du temps de l'implication potentielle de l'insight dans la survenue de l'addiction : évolutions parallèles de l'état interne, de l'insight et des insight $S$ et insight P. En haut, état interne (ligne verte) transitoirement excité lors de la première prise de drogue. Au milieu, déficit d'insight (ligne noire) plus important avant développement de la pathologie. En bas, composante somatosensorielle de l'insight (ligne rose) : gain d'insight $\mathrm{S}$ en lien avec l'exacerbation des sensations. En bas, composante psychique de l'insight (ligne bleue) : dégradation d'insight $\mathrm{P}$ lors de la mise en place d'un consommation compulsive de la drogue en dépit de ses conséquences néfastes.

\section{Discussion \\ Discussion}

Pr Houillon - Au cours de cette communication viennent d'être évoqués les liens entre le déficit d'insight et les addictions avec une argumentation des plus convaincantes. Et puisque l'auteur a aussi fait allusion aux états d' «hyper-insight», j’aimerais savoir s’il considère que ceux-ci ont des liens tout aussi étroits avec les comportements addictifs. Cela dans la mesure où l'hyper-insight peut résulter d'une recherche de compensation à un 
sentiment d'insuffisance, capable à son tour d'induire, par substance « ad hoc » substitutive interposée, accoutumance et dépendance. Dans le même contexte, celui au fond du maintien d'un état de vigilance satisfaisant, voire surdimensionné, n’y a-t-il pas à établir encore une relation avec le trouble obsessionnel compulsif qui a aussi pour fonction une «stabilisation des attentes », autrement dit la défense de l'insight propre au sujet, à la structure de sa personnalité, à ce qu'il a à vivre et à la perception qu'il a de ce qu'il est et de ce qu'il a à faire.

Réponse du Rapporteur - La question de l'hyper-insight est effectivement très pertinente dans le contexte des addictions, et certainement dans celui du développement de ces nouvelles générations de psychotropes dits « cognitive enhancers ». L’hyper-insight est effectivement supposé jouer un rôle non négligeable dans le modèle étiologique de l’addiction aux stimulants et même dans celui des troubles obsessionnels compulsifs. Commençons par l'exemple de l'addiction : la recherche d'hyper-insight peut être considérée comme substrat psychologique au trait de recherche de sensations où le sujet souffrant d'un déficit d'insight somatosensoriel cherche, à travers une stimulation extrême, à « se rencontrer ». Cette rencontre, il la vivrait dans la plénitude uniquement par les effets iatrogènes des stimulants. Ainsi, le «high» et "l'hyper-awareness » vécus par les consommateurs de stimulants pourraient refléter un hyper-insight qui, expérimenté par des sujets sans déficit préalable, ne revêtirait pas de dimension pathologique, mais serait un moteur de l'addiction chez les autres.

Dans le contexte du trouble obsessionnel-compulsif, l’obsession, cette pensée intrusive associée à une dimension anxieuse, pourrait être suggérée comme épisode d’hyper-insight. En effet, l'obsession serait, chez les patients ayant un déficit d'insight somatosensoriel ou psychique les empêchant d'attribuer une valence anxieuse à leur état interne ou aux stimuli externes aversifs, une médication de ce déficit à travers un processus d’isolement du déséquilibre, à visée de contrôle avec la compulsion. Ainsi l'hyper-insight spécifique de l'anxiété dans le TOC ne serait que transitoire et principalement de valence somatosensorielle. En effet, l'objet de l'obsession par essence n'est pas la cause réelle du désordre anxieux. Le déficit d’insight psychique s’exprimerait là dans la mauvaise attribution des causes de l'obsession et de la compulsion.

Pr Lafont - Comment situer, par exemple, les deux « consciences du trouble » que sont la presbyophrénie et la mélancolie dans le schéma de modélisation proposé par l’intervenant? 
Réponse du Rapporteur - L’échelle d’insight proposée mérite effectivement d’être confrontée à la clinique. Tout d'abord, l'anamnèse tiendrait une place indispensable à ce genre de réponse. Mais on peut envisager plusieurs combinaisons d’altérations qui résulteraient en un déficit de représentation temporospatiale et des fausses reconnaissances, qui sont les principales caractéristiques de la démence de Wernicke. L’affection concernerait principalement l'insight psychique, tant au niveau de la représentation de l'état psychique qu'au niveau de la conscience de cette représentation, intrinsèquement liée à la mémoire autobiographique. Pour le deuxième exemple, la mélancolie, deux cas de figure peuvent être envisagés. D’abord, un déficit avec polarité somatosensorielle, pour lequel nous rejoindrions l'hypothèse des marqueurs somatiques de Damasio, en suggérant que le patient ne ressent plus de bonheur ou de plaisir parce qu'il n'en a plus les signaux somatosensoriels. Ce faisant, tant sa représentation somatosensorielle que la conscience de cette représentation altéreraient les équivalents d'intégration au niveau de l'insight psychique. L'inverse pourrait également être vrai, c'est-à-dire qu'un déficit de représentation d'insight psychique amènerait le patient à avoir une conscience altérée de son état, le conduisant à la mélancolie. Évidemment, les deux mécanismes décrits ne sont pas mutuellement exclusifs et peuvent se superposer.

Pr Laxenaire - Je voulais revenir sur votre distinction entre insight somato-sensoriel et insight psychique en vous demandant si, plutôt que de « bidirectionnel », il ne s'agissait pas de hiérarchie ou même de subordination entre la première et la seconde ?

Je peux en effet avoir une connaissance de ce qui se passe dans mon corps et lui donner des réponses différentes: pour tout dire, normale ou pathologique. D’où vient la différence entre les deux réponses ? N'y aurait-il pas un autre niveau où l'insight serait juge de l'insight et lui conférerait des significations conduisant à la distinction fondamentale entre le normal et le pathologique?

Réponse du Rapporteur - Vous avez raison. Comme la structure générale du modèle le représente explicitement, il y a effectivement d'autres relations hiérarchiques entre insight somatosensoriel et insight psychique que celles fondées sur l'interconnexion bidirectionnelle de chacun des niveaux d'intégration.

Même si l'on concède que l'insight psychique restera le dernier niveau d'intégration de ce processus complexe, le modèle suggère une inter-relation entre insight somatosensoriel et insight psychique. Ainsi, les niveaux primaires de l'élaboration de l'insight somatosensoriel peuvent, sans que l'individu n'en soit conscient, altérer le processus de l’insight psychique. 
Les réponses différentes à la perception consciente que l'individu a de ses manifestations corporelles peuvent être l'expression : soit d'un processus dysfonctionnel de conscience de la représentation de soi, par traitement altéré des informations normales issues des couches inférieures des processus psychique et somatosensoriel; soit du traitement normal d'informations inexactes provenant des premières couches d'intégration de l'insight somatosensoriel.

Ainsi, ce modèle ne propose pas de résoudre la question de l'insight juge de lui-même mais bien la nature de l'altération de ce jugement, ainsi que des potentielles erreurs d'attribution associées, qui sont intrinsèques au caractère pathologique du processus en cours dans les affections psychiatriques. 